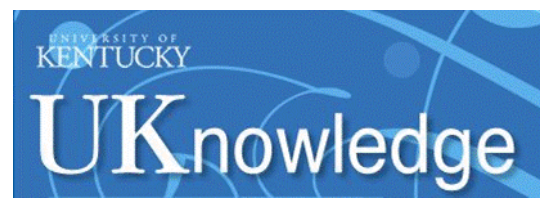

University of Kentucky

UKnowledge

6-1-2010

\title{
Luminosity-variation Independent Location of the Circum-Nuclear, Hot Dust in NGC 4151
}

Jorg-Uwe Pott

Max-Planck-Institut für Astronomie, Germany

Matt A. Malkan

University of California

Moshe Elitzur

University of Kentucky, moshe@pa.uky.edu

Andrea M. Ghez

University of California

Tom M. Herbst

Max-Planck-Institut für Astronomie, Germany

See next page for additional authors

Follow this and additional works at: https://uknowledge.uky.edu/physastron_facpub

Part of the Astrophysics and Astronomy Commons, and the Physics Commons

Right click to open a feedback form in a new tab to let us know how this document benefits you.

\section{Repository Citation}

Pott, Jorg-Uwe; Malkan, Matt A.; Elitzur, Moshe; Ghez, Andrea M.; Herbst, Tom M.; Schödel, Rainer; and Woillez, Julien, "Luminosity-variation Independent Location of the Circum-Nuclear, Hot Dust in NGC 4151" (2010). Physics and Astronomy Faculty Publications. 194.

https://uknowledge.uky.edu/physastron_facpub/194

This Article is brought to you for free and open access by the Physics and Astronomy at UKnowledge. It has been accepted for inclusion in Physics and Astronomy Faculty Publications by an authorized administrator of UKnowledge. For more information, please contact UKnowledge@lsv.uky.edu. 


\section{Luminosity-variation Independent Location of the Circum-Nuclear, Hot Dust in NGC 4151}

\section{Digital Object Identifier (DOI)}

http://dx.doi.org/10.1088/0004-637X/715/2/736

\section{Notes/Citation Information}

Published in The Astrophysical Journal, v. 715, no. 2, p. 736-742.

(C) 2010. The American Astronomical Society. All rights reserved.

The copyright holder has granted permission for posting the article here.

\section{Authors}

Jorg-Uwe Pott, Matt A. Malkan, Moshe Elitzur, Andrea M. Ghez, Tom M. Herbst, Rainer Schödel, and Julien Woillez 


\title{
LUMINOSITY-VARIATION INDEPENDENT LOCATION OF THE CIRCUM-NUCLEAR, HOT DUST IN NGC 4151
}

\author{
Jorg-Uwe Pott ${ }^{1,2,3}$, Matt A. Malkan ${ }^{2}$, Moshe Elitzur $^{4}$, Andrea M. Ghez ${ }^{2,5}$, Tom M. Herbst ${ }^{1}$, Rainer Schödel ${ }^{6}$, \\ AND JULIEN WOILLEZ ${ }^{3}$ \\ ${ }^{1}$ Max-Planck-Institut für Astronomie, Königstuhl 17, D-69117 Heidelberg, Germany; jpott@mpia.de, herbst@mpia.de \\ ${ }^{2}$ Division of Astronomy \& Astrophysics, University of California, Los Angeles, CA 90095-1547, USA; malkan@ astro.ucla.edu, ghez@astro.ucla.edu \\ ${ }^{3}$ W. M. Keck Observatory, California Association for Research in Astronomy, Kamuela, HI 96743, USA; jwoillez@keck.hawaii.edu \\ ${ }^{4}$ Department of Physics and Astronomy, University of Kentucky, Lexington, KY 40506-0055, USA; moshe @ pa.uky.edu \\ ${ }^{5}$ Institute of Geophysics and Planetary Physics, University of California, Los Angeles, CA 90095-1565, USA \\ ${ }^{6}$ Instituto de Astrofisica de Andalucia-CSIC, Camino Bajo de Huetor 50, 18008 Granada, Spain; rainer@iaa.es \\ Received 2009 December 22; accepted 2010 March 22; published 2010 May 3
}

\begin{abstract}
After recent sensitivity upgrades at the Keck Interferometer (KI), systematic interferometric $2 \mu \mathrm{m}$ studies of the innermost dust in nearby Seyfert nuclei are within observational reach. Here, we present the analysis of new interferometric data of NGC 4151, discussed in context of the results from recent dust reverberation, spectrophotometric, and interferometric campaigns. The complete data set gives a complex picture, in particular the measured visibilities from now three different nights appear to be rather insensitive to the variation of the nuclear luminosity. KI data alone indicate two scenarios: the $K$-band emission is either dominated to $\sim 90 \%$ by size scales smaller than $30 \mathrm{mpc}$, which falls short of any dust reverberation measurement in NGC 4151 and of theoretical models of circum-nuclear dust distributions. Or contrary, and more likely, the $K$-band continuum emission is dominated by hot dust $(\gtrsim 1300 \mathrm{~K})$ at linear scales of about $50 \mathrm{mpc}$. The linear size estimate varies by a few tens of percent depending on the exact morphology observed. Our interferometric, deprojected centro-nuclear dust radius estimate of $55 \pm 5 \mathrm{mpc}$ is roughly consistent with the earlier published expectations from circum-nuclear, dusty radiative transfer models, and spectro-photometric modeling. However, our data do not support the notion that the dust emission size scale follows the nuclear variability of NGC 4151 as an $R_{\text {dust }} \propto L_{\text {nuc }}^{0.5}$ scaling relation. Instead variable nuclear activity, lagging, and variable dust response to illumination changes need to be combined to explain the observations.
\end{abstract}

Key words: galaxies: individual (NGC 4151) - galaxies: nuclei - galaxies: Seyfert - techniques: interferometric

Online-only material: color figures

\section{INTRODUCTION}

Nearby active galactic nuclei (AGNs) are rosetta stones for the understanding of the astrophysics close to an actively accreting supermassive black hole (SMBH). Sub-parsec resolution is required to identify answers to the key questions of AGN physics. (1) How does galactic material flow down to the accretion disk to feed luminosities close to the Eddington limit? (2) Which role do outflows and jets play in the energetic interconnection between the AGN and its host? (3) Are AGNs of different luminosities intrinsically similar? Studying the spectral energy distribution (SED) of quasars and type 1 AGN from the optical to the far-infrared reveals remarkably similar SED shapes, suggesting similar physics (Edelson \& Malkan 1986; Sanders et al. 1989; Kobayashi et al. 1993; Riffel et al. 2006). Therefore, resolving astrophysical phenomena in nearby AGN enables to better understand farther, unresolved nuclei, and to gauge correlations between nuclear luminosity and properties of the surrounding circum-nuclear environment. Being one of the brightest AGN on the sky, NGC 4151 reveals this unique potential of nuclei closer than a few tens of Mpc in numerous publications.

In this article, we concentrate on the origin of the near-infrared (NIR) emission of NGC 4151 and its relation to the nuclear luminosity. NIR AGN emission is characterized by a steep decline from optical wavelengths down to about $1 \mu \mathrm{m}$, and on the long wavelength side by the quick rise of a separate IR emission bump peaking at about $3 \mu \mathrm{m}$ (Edelson \& Malkan 1986;
Kobayashi et al. 1993). The origin of this NIR excess has been discussed throughout the past four decades (probably one of the first were Pacholczyk \& Weymann 1968; Rees et al. 1969). While it is not in question that the original power originates in the accretion disk, it is uncertain if the NIR emission excess derives directly from nuclear emission processes, or if mostly dust outside the broad line region (BLR) re-radiates the nuclear emission (Edelson \& Malkan 1986; Edelson et al. 1988; Barvainis 1987, 1992). The proper measurement of the nuclear near-infrared emission (and its discrimination from dustre-processed light) is crucial to derive the intrinsic SED of accreting SMBHs in the centers of galaxies and to understand the radiative processes involved.

Even at the relatively detailed linear scale of NGC 4151 ( $~ 82 \mathrm{mpc} \mathrm{mas}^{-1}$, footnote "a" in Table 3$)$, the accretion disk itself and the surrounding BLR are too small to be spatially resolved by the current generation of optical or infrared telescopes or interferometers (Bentz et al. 2006). However, the circumnuclear dust distribution is now within reach of observations with infrared telescope arrays at sub-50 mas angular resolution (Swain et al. 2003; Wittkowski et al. 2004; Jaffe et al. 2004). Mid-infrared data of the Very Large Telescope interferometer typically find in Seyfert nuclei $10 \mu \mathrm{m}$ emission sizes of a few parsecs (Tristram et al. 2009; Burtscher et al. 2009; Raban et al. 2009). Dust plays an exceptional role among the circum-nuclear components. It is not only assumed to significantly contribute to the near-to-mid-infrared continuum radiation of an AGN. Also, a non-spherically symmetric dust distribution is widely 
Table 1

Observing Log

\begin{tabular}{lcccccc}
\hline \hline Target & Date (UT) & $\begin{array}{c}\text { H.A. }^{\mathrm{a}} \\
(\mathrm{hr})\end{array}$ & $\begin{array}{c}u, v^{\mathrm{b}} \\
(\mathrm{m})\end{array}$ & $\begin{array}{c}\text { Proj. B } \\
(\mathrm{m}, \operatorname{deg}(\text { EofN)) }\end{array}$ & $\begin{array}{c}\text { Calibrators } \\
(\text { from Table 2) }\end{array}$ & $\begin{array}{c}V^{2} \\
(\mathrm{calib} .)^{\mathrm{c}}\end{array}$ \\
\hline NGC 4151 & 2008 Dec 15 & -1.3 & $(56.4,51.6)$ & $(76.4,47.5)$ & 1,2 & 0.85 \\
& 2008 Dec 15 & -1.1 & $(56.0,54.0)$ & $(77.8,46.0)$ & 1,2 & 0.84 \\
\hline
\end{tabular}

Notes.

${ }^{\text {a }}$ Hour angle.

${ }^{\mathrm{b}}$ The $u$-, $v$-coordinates, given here in meters, are the baseline length (B) projected onto the line of sight. $u$ points east, $v$ points north. They are equivalent to polar values of the projected baseline, given in the next column in meters, and degrees east of north.

${ }^{\mathrm{c}}$ The absolute calibration accuracy is at least 0.03 , which is shown in Figure 1 and should be used if comparing these values with results from different nights and observing campaigns. The differential intranight visibility precision is $0.01-0.015$, based on the statistical scatter of the measurements.

assumed to explain the type $1 / 2$ dichotomy of AGN emission line spectra. Radiative transfer models of clumpy distributions of dust clouds extending out of the equatorial plane are currently the favored explanation of the steadily increasing amount of observational data (Barvainis 1987, 1992; Schartmann et al. 2005; Hönig et al. 2006; Nenkova et al. 2008a, 2008b; Hönig \& Kishimoto 2009). In the following, we refer to the dust distribution as the torus; keeping in mind that its detailed morphology is rather uncertain, it might not resemble closely a smooth torus.

It is currently an open debate, how the findings and models at MIR wavelengths connect to the innermost hot dust, which is expected to contribute to the continuum emission at $2 \mu \mathrm{m}$. Mor et al. (2009) argue for a spatially and chemically distinct inner component, based on 2-35 $\mu \mathrm{m}$ spectra. Also, the interferometric MIR data of NGC 4151 appear to reject that the nuclear flux at $10 \mu \mathrm{m}$ is dominated by the outer, cooler part of the same structure, which dominates the $2 \mu \mathrm{m}$ continuum (Burtscher et al. 2009). Such results discourage simple attempts to unify the dust emission structures around AGNs, and suggest multicomponent models, or at least a significant change of dust composition and grain size distribution with the radial distance to the central engine (Schartmann et al. 2005; Kishimoto et al. 2009b).

We report in this paper on the first results of a new campaign using the Keck Interferometer (KI) to add observational constraints to the origin of the NIR continuum of NGC 4151. The $85 \mathrm{~m}$ baseline and the sensitivity of the KI are adequate to resolve the linear distances of order 30-200 mpc around NGC 4151 which matches the theoretically calculated dust sublimation radii for this source. Recent sensitivity improvements of the KI (Wizinowich et al. 2006; Ragland et al. 2008) 7 enabled us to repeat the early Swain et al. KI measurement, although the variable nucleus of NGC 4151 has been at a significantly fainter state during the time of observation. After a description of the observations (Section 2), we discuss our findings and the implications on the interpretation of the combined visibility data set (from Swain et al. 2003; Kishimoto et al. 2009a, and the here presented observations) in Section 3. We concentrate our discussion on issues left open by the previous high resolution NIR observations of the AGN: (1) is the $K$-band emission rather dominated by unresolved accretion disk emission (as favored by Swain et al. 2003), or by resolved circum-nuclear dust emission (Kishimoto et al. 2009a)? (2) How closely related are accretion disk luminosity and circum-nuclear dust location in NGC 4151 (Koshida et al. 2009)? (3) Is the currently described scatter

\footnotetext{
7 Current KI performance numbers are given at the Web site of NASA Exoplanet Science Institute (NEXSCI):

http://nexsci.caltech.edu/software/KISupport/v2/v2sensitivity.html.
}

Table 2

Properties of the Interferometric Calibrator Stars Used for Calibration of the Instrumental Transfer Function During Data Reduction

\begin{tabular}{lcccc}
\hline \hline No. & Calibrator & $V / H / K^{\mathrm{a}}$ & Spec. Type $^{\mathrm{a}}$ & Ang. Diameter (mas) $^{\mathrm{b}}$ \\
\hline 1 & HIP58819 & $10.9 / 8.5 / 8.4$ & K0III & $0.11 \pm 0.03$ \\
2 & HD109691 & $8.9 / 8.9 / 8.9$ & A0V & $0.04 \pm 0.02$ \\
\hline
\end{tabular}

Notes. Calibrator stellar diameters significantly smaller than 0.5 mas are unresolved by the KI. The statistical errors given here for the bolometric diameter fit to a blackbody likely slightly underestimate systematic errors of the NIR diameter of stars, but even 0.2 mas uncertainties in the diameter would not change the visibility calibration.

${ }^{a}$ From Simbad.

${ }^{b}$ Bolometric diameter fit from the NexsCI getCal tool.

between direct interferometric and indirect reverberation measurements of the torus size a systematic offset reflecting that the respective method probes slightly different dust reservoirs, or does the scatter rather reflect the currently achievable accuracy of either method in deriving the actual dust location (Koshida et al. 2009; Kishimoto et al. 2009a)? The concluding remarks in Section 4 summarize our current results and give a brief outlook on the project.

\section{OBSERVATIONS}

The $85 \mathrm{~m}$ baseline of the KI is oriented $38^{\circ}$ east of north. ${ }^{7}$ We used the $K^{\prime}$-band $(2-2.4 \mu \mathrm{m}) V^{2}$ continuum mode. All data shown here are from the white-light channel of the beam combiner, observed at an effective wavelength of 2.18 $\mu \mathrm{m}$. The observations were conducted on 2008 December 15 (UT), details appear in Table 1. We followed standard observing and data reduction procedures (see Section 3.2 in Colavita et al. 2003, and references therein). KI data are provided to the observer in a semi-raw state, and still require estimation and correction for the system visibility (or visibility transfer function), which is estimated by observing unresolved calibrators, close in space and time (typical numbers for close are $\lesssim 10^{\circ}$ and $\lesssim 0.5 \mathrm{hr}$ ). We chose calibrators of a $K$-band magnitude similar to the target, to avoid a flux bias in the calibrated visibilities $\left(V^{2}\right.$; Table 2$)$. Our typical scanlength, leading to one visibility point, is $200 \mathrm{~s}$.

A reliable data calibration is indicated by stable system visibility and the flux ratios between both telescope beams over the night. Our observation was conducted at a visible seeing of 0 '.5. To estimate and apply the system visibility, we used the wb/nbCalib-software suite by Nexscr. We use the standard deviation over the about 25 individual visibility estimations, which make up the $200 \mathrm{~s}$ scan, as a first estimate 


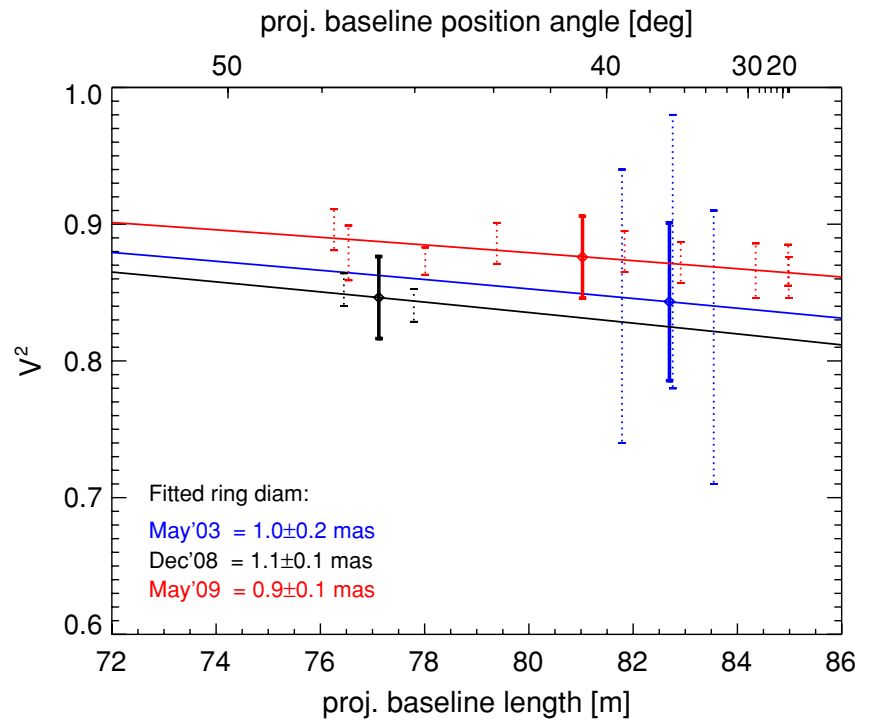

Figure 1. Calibrated visibility data from our observations (black), and from two other measurements (blue, red: Swain et al. 2003; Kishimoto et al. 2009a, respectively), plotted vs. the projected baseline length. The dotted error bars show the individual data points, with their estimated uncertainties. The three larger solid error bars show the mean $V^{2}$ result of each campaign and the adequate accuracy bias of at least 0.03 . The smaller statistical errors reflect the differential precision of the observations within a night, the larger accuracy bias needs to be taken into account if absolute size scales are fitted, and if observations from different nights are compared. $\chi^{2}$ minimized face-on ring models are overplotted, the best-fit diameters are given. A compact flux contribution from the AGN on the order of up to $25 \%$ does not alter the results significantly (see Table 3 for various model-fit results). The total $K$-band flux shown comprises the flux contributions from the accretion disk and from the circum-nuclear dust (see Section 2 for flux calibration details)

(A color version of this figure is available in the online journal.)

of the uncertainties of each data point. The resulting statistical noise in the calibrated visibility measurement is $0.01-0.015$, proving the good observing conditions of the night. This gives the differential intranight visibility precision, and can be used to check for visibility slopes with respect to changes of the baseline projection. Long-term monitoring of KI data against known binary orbits shows that the absolute calibration accuracy of the data from night to night is at least $0.03 .{ }^{7}$ The difference between the intranight precision and internight accuracy is visualized in Figure 1 . We use the 0.03 uncertainty to derive absolute emission sizes (Table 3) and to check for flux-induced size variations (Figure 2).

The NIR flux of NGC 4151 is variable, and a contemporaneous total flux measurement is needed in our discussion of the

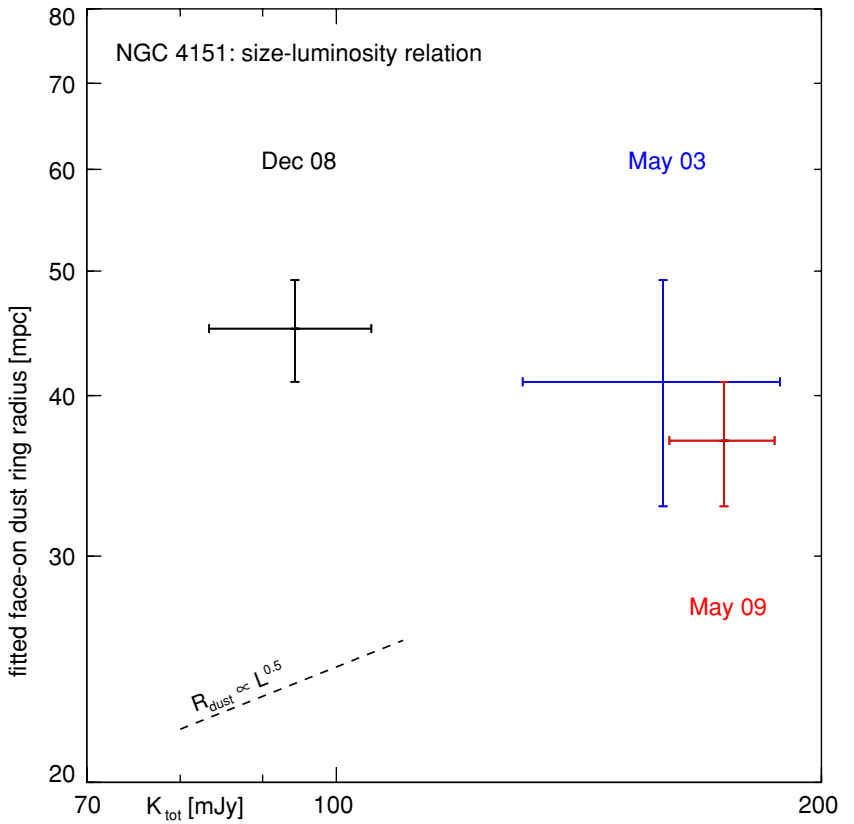

Figure 2. Dust ring radii derived from fitting a face-on ring to the calibrated visibility data. Color coding matches Figure 1 . In the lower left, an $L^{0.5}$ dependence is shown for comparison. Note, that the plotted radii take into account neither projection effects nor variable nuclear flux contributions. An inclined dust ring would have larger centro-nuclear radii. A nuclear flux contribution, varying between $10 \%$ and $25 \%$, could explain the apparent slope of the three data points, as discussed in Section 3.2.

(A color version of this figure is available in the online journal.)

data, to properly compare our visibilities with data from other observing epochs. A direct flux calibration of the total photon counts detected by the interferometric camera (called FATCAT) needs to reflect that FATCAT is fed by single-mode fibres. The fibres realize a spatial filtering of $\sim 50$ mas on sky to reduce the atmospheric phase noise. Since this quasi-aperture is on the order of the Keck diffraction limit at $2.2 \mu \mathrm{m}$, this introduces a Strehl dependence of the $K$-band flux measurements. The data show that the Strehl on NGC 4151 is systematically lower than on the calibrator stars, because the stars are significantly bluer then the red AGN and deliver more photons to the visible wavefront sensor (WFS; note that we matched calibrator stars and AGN in the infrared brightness to get good visibility accuracy). Furthermore, the WFS probably detects some extended flux from the host galaxy.

To account for this differential Strehl, the raw data of the AGN are multiplied by 1.45 , before the flux calibration of the

Table 3

Model-fit Parameters Consistent With the Data

\begin{tabular}{lcccc}
\hline \hline Model & $F R_{\text {nuc }}^{K}$ & $($ mas $)$ & $R_{\text {dust }}$ & Type of Dust Distribution \\
\hline 1 & 0.9 & $\gtrsim 5$ & $\gtrsim 400$ & Overresolved \\
2 & 0.25 & $0.55 \pm 0.05$ & 45 & Resolved ring \\
2 & 0 & $0.6 \pm 0.05$ & 50 & Resolved ring \\
2 & 0.25 & $0.45 \pm 0.05$ & 35 & Resolved Gaussian \\
2 & 0 & $0.5 \pm 0.05$ & 40 & Resolved Gaussian \\
3 & 0 & $0.75 \pm 0.05$ & 60 & Resolved ring, 45 inclined, \\
& & & & Minor axis co-aligned with the KI baseline
\end{tabular}

Notes. The varying fit results of the ring models 2 and 3 reflect projection effects and represent general lower and upper limits on the intrinsic $R_{\text {dust }}$ (Section 3.1)

${ }^{\text {a }}$ From NED: cosmology-corrected redshift of 0.00414 against the $3 \mathrm{~K}$ microwave background reference frame results in angular-size distance of $16.9 \mathrm{Mpc}$ and $82 \mathrm{mpc} / \mathrm{mas}$, respectively, with $H_{0}=73 \mathrm{~km} \mathrm{sec}^{-1} \mathrm{Mpc}^{-1}, \Omega_{\text {matter }}=0.27$. 
raw data against the calibrator stars. This empirical correction factor for the differential Strehl (between stars and AGNs) of 1.45 appears to be stable over time, within the precision of the flux count estimation. It fits to the data of the two campaigns targeting NGC 4151, where quasi-simultaneous $K$-band photometry from a separate telescope is available: Kishimoto et al. (2009a) and Swain et al. (2003). While the former publish quasi-contemporaneous UKIRT photometry, we re-analyzed the archived KI data of the latter paper and compared the FATCAT photometry against the simultaneous MAGNUM single telescope photometry, as presented by Koshida et al. (2009). To cross-check that by applying the correction factor, we account properly for the loss of light due to the spatial filtering of our $K$-band data, we also flux calibrated the spatially unfiltered $1.6 \mu \mathrm{m}(H)$-band flux of the tip-tilt imager next to FATCAT, which feeds a tip-tilt stabilizing loop during KI measurements. Both this $H$-band flux $(50 \mathrm{mJy})$ and the corrected $K$-band flux (95 mJy) fit the corresponding values in Kishimoto et al. (2009a), with the same flux offset of a factor of 1.9, which we account to intrinsic flux variability. In Figure 2, we used for the $K$-band photometry of the three visibility measurements the corrected FATCAT photometry for the Swain et al., and our data, and the UKIRT photometry from Kishimoto et al. 2009a, 2009b, together with the respective photometric uncertainties. The flux contamination of the host galaxy in the $K$ band is with about $1 \%$ neglectable (Kishimoto et al. 2009a). The flux correction factor of 1.45 for the FATCAT $K$-band data of NGC 4151 seems to be stable over the years, and therefore not too sensitive to the actual observing conditions, as long as a similar calibration scheme is used. This is probably due to the fact that in case of poor, unstable adaptive optics performance the interferometric measurement would not work efficiently. But the correction factor should not be applied to future data sets without careful checking of the effective differential Strehl between calibrator and target during the respective observation. In particular, the correction factor should be re-estimated for KI observations of different targets.

\section{DISCUSSION OF THE RESULTS}

The calibrated visibilities are plotted in Figure 1 together with previously published data from Swain et al. (2003) and Kishimoto et al. (2009a). It is apparent that the different interferometry campaigns measured very similar visibilities, despite of the significantly different total flux measured (Figure 2). By fitting models, we focus on three different properties of the size constraints which can be derived from the visibilities: the amount of unresolved flux, the size of the resolved flux emission structure, and the deprojection of this size. Our models consist of a variable fractional amount of (unresolved) nuclear emission $\left(F R_{\text {nuc }}^{K}=0-1\right)$ along with resolved (overresolved) emission at (beyond) centro-nuclear radii $R_{\text {dust }}$ (Figure 3 ). We simply refer to the extended component as dust, being the most likely origin of it.

\subsection{Modeling the Dust Emission}

Model 1. Already a single visibility below unity shows that some fraction of the flux is resolved by the interferometer. This corresponds to $F R_{\text {nuc }}^{K}<1$. The most compact brightness distribution, still consistent with the data, would put $F R_{\text {nuc }}^{K} \sim$ $90 \%$ of the flux at radii smaller than 0.35 mas ( $<30 \mathrm{mpc})$ below the resolution limit of the KI ("point source"), and the remainder of the flux outside of $R_{\text {dust }} \sim 400$ mpc to be completely resolved

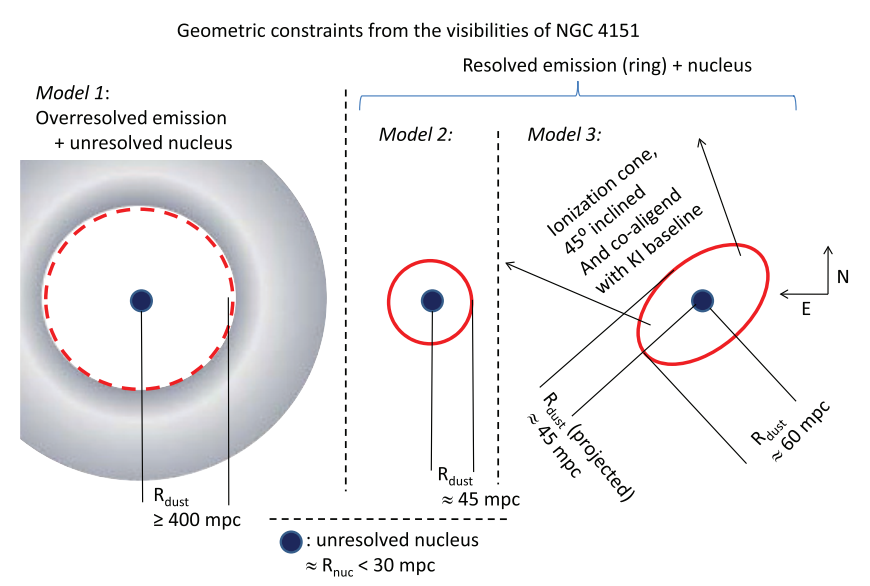

Figure 3. Sketches (not to scale) of the geometric constraints on the extended $K$-band emission of NGC 4151, seen through the 50 mas spatial filter of the Keck interferometer. Details are given in Section 3.1. Note that the overresolved emission in model 1 might be diffuse, or more spherically than drawn, but the emission needs to origin from centro-nuclear radii larger than $400 \mathrm{mpc}$ to be completely resolved out, and to not contribute to the $V^{2}$ budget.

(A color version of this figure is available in the online journal.)

out. Since radii of about $30 \mathrm{mpc}$ are significantly smaller than the dust sublimation radius in NGC 4151, even for large graphite grains (see the recent discussions in Kishimoto et al. 2007; Nenkova et al. 2008b), this scenario would suggest that the $K$-band emission is dominated by hot, and potentially partially non-thermal nuclear emission from the innermost accretion disk zone.

But the derivation of $F R_{\text {nuc }}^{K}$ from a few visibilities is degenerate. Reducing $F R_{\text {nuc }}^{K}$ requires only to shrink $R_{\text {dust }}$ to stay consistent with the data. The visibility alone does not constrain $F R_{\text {nuc }}^{K}$ any further than being $\leqslant 0.9$. However, recent spectrophotometric and NIR-polarimetric data appear to limit $F R_{\text {nuc }}^{K}$ to less than $25 \%$. In these studies, the central $K$-band flux appears dominated by moderately warm thermal emission $(\sim 1500 \mathrm{~K})$, likely from circum-nuclear dust clouds (see the detailed discussions and references of the observational support of dust being the dominating origin of the $K$-band emission in Seyfert 1 nuclei in Barvainis 1987; Riffel et al. 2009; Kishimoto et al. 2009b, 2009a). Since our KI photometry and visibilities from the December 2008 run are consistent with these earlier findings, we assume in the following $F R_{\text {nuc }}^{K} \lesssim 0.25$, which means that both total and correlated fluxes are dominated by extended emission with an emission size similar to the angular resolution of the interferometer. Such resolved emission would result in decreasing visibilities with increasing baseline length. Indeed such a trend is marginally apparent in both new data sets with high intranight differential visibility precision (December 2008 and May 2009 in Figure 1).

Model 2. This model aims at estimating the order of magnitude of $R_{\text {dust }}$. To translate visibilities into size scales, a simple model of the extended emission is required. Gaussians and rings fit the data equally well and give the similar $R_{\text {dust,circ }}$ of $\sim 45 \mathrm{mpc}\left(0.5 \times \mathrm{FWHM} \approx R_{\text {ring }}=0.55 \pm 0.1 \mathrm{mas}\right.$; Figure 1 and Table 3$)$. This size estimate is largely unaffected by an $F R_{\text {nuc }}^{K} \lesssim 25 \%$, or by the radial extension of the ring as long as the ring radius is larger than its extension. The key difference between Gaussian and ring models is that the Gaussian not only defines a size scale, but at the same time it constrains the emitting area. The fitted FWHM translates into a solid angle of $0.92 \mathrm{mas}^{2}$. However, a brightness temperature calculation rejects a smooth Gaussian-like emission surface profile. Assuming 
a blackbody temperature of $1300 \mathrm{~K}$, this surface would result in about $500 \mathrm{mJy}$. This is about a factor of 2.5-10 larger than the measured dusty $K$-band emission of NGC 4151, depending on its status of nuclear activity (Figure 2). If the dust emission is optically thick, this discrepancy means that the radiating surface area is significantly smaller than the solid angle of the fitted Gaussian. If we were to shrink the FWHM of the Gaussian to match the observed fluxes, the resulting increased visibilities would overshoot over the data. Higher dust temperatures would strengthen this argument against a smooth Gaussian brightness distribution. Thus, we conclude that the blackbody component, apparent in the spectro-photometry (e.g., Riffel et al. 2009), is not produced by an approximately spherical, smooth dust distribution (which would resemble a Gaussian in projection). The planar geometry of the ring model seems more reasonable. This matches the expectation for observing a type 1 AGN. Optically thick clouds, as used in modern torus models, would reduce the radiating surface as well (without changing the $R_{\text {dust }}$ estimate). The ring morphology also appears reasonable due to the lack of significant extinction toward the very nucleus (Lacy et al. 1982).

Model 3. The $R_{\text {dust,circ }}$ estimate from the previous paragraph assumes a face-on, circular dust distribution. However, given the single KI baseline, the size constraints of KI data are primarily one dimensional along the position angle (P.A.) of the baseline (about $40^{\circ}$ ). The resolved source could be substantially larger along the orthogonal (SE-NW) direction. In fact, the KI baseline P.A. roughly co-aligns with the ionization cone axis of symmetry of NGC 4151. Das et al. (2005) and Riffel et al. (2009) report position, opening, and line-of-sight inclination angles of the cone to be $\sim 60^{\circ}, \sim 70^{\circ}$, and $\sim 45^{\circ}$. If the circum-nuclear dust, seen as extended component by the interferometer, is responsible for the formation of the ionization cone, the opening angle would translate into a torus half-height of 0.7 times the inner radius. Such a geometry matches the thin ring model, if the extended $K$-band emission is dominated by the hottest dust exposed to direct nuclear illumination. If the KI and the projected minor axis were exactly co-aligned, the size estimate from the previous face-on model $\left(R_{\text {dust,circ }} \sim 45 \mathrm{mpc}\right)$ needs to be corrected for the inclination angle to $R_{\text {dust,incl }} \sim 60 \mathrm{mpc}$ (see Table 3 ). The P.A. of the inner radio jet, a possibly better constraint on the AGN axis of symmetry, lies with a P.A. of $\sim 80^{\circ}$ within this ionization cone (Mundell et al. 2003), but slightly off the KI P.A. Summarizing, the intrinsic three-dimensional inner torus radius $R_{\text {dust }}$ is constrained to $R_{\text {dust, circ }} \leqslant R_{\text {dust }} \leqslant R_{\text {dust,incl }}$. Taking into account the orientation constraints of radio jet and ionization cone, we estimate $R_{\text {dust }}=55 \pm 5 \mathrm{mpc}$. The significant inclination of the nuclear axis of symmetry, indicated by the inclination of the ionization cone, is supported by the time-variable, spectral classification of NGC 4151 as Seyfert 1.5-1.8 (Shapovalova et al. 2008).

\subsection{On the Luminosity Dependence of the Torus Size}

In general, both $V$-to- $K$-continuum reverberation and NIR interferometric measurements of nearby AGN appear to confirm an $L^{0.5}$ dependence of the extended $K$-band emission size on the nuclear illumination luminosity (Suganuma et al. 2006; Kishimoto et al. 2009a). In addition, the NIR SED bump of AGN can be fitted consistently with a blackbody profile of about $1500 \mathrm{~K}$, for AGN samples covering orders of magnitude in nuclear power (Kobayashi et al. 1993). These are strong arguments for the $K$ band being dominated by thermal dust emission close to the sublimation limit. The strong nuclear emission variability of NGC 4151 by several $100 \%$ on yearly timescales makes it an ideal target to do the next step and to study the relation between the nuclear emission and the dust emission size in detail. Sequential dust reverberation studies in several AGN did find changes of the response delay time, suggesting a change of $R_{\text {dust }}$ in reaction to different nuclear illumination (Barvainis 1992; Koshida et al. 2009). However, given an $R_{\text {dust }}$ of several tens of mpc, it would require unreasonable fast dust bulk motion (at $0.1 c$ ) to follow the luminosity changes according to an $L^{0.5}$ law. Therefore, cyclic dust grain destruction and formation was suggested to explain both the observed infrared flux variations (Barvainis 1992) and the large scatter and relatively poor $L^{0.5}$ dependence in the dust reverberation size estimates of NGC 4151 (Koshida et al. 2009).

For the first time, we can compare these claims of a changing $R_{\text {dust }}$ against a multi-epoch visibility data set, taken at different brightness states (Figure 2) of NGC 4151. Assuming a simple $L^{0.5}$ scaling, the flux variation between the two new data sets $\left(F_{\mathrm{K}, \text { May } 09} / F_{\mathrm{K} \text {, Dec } 08}=1.9 \pm 0.3\right)$ would result in a size increase by a factor of $1.4 \pm 0.1$. Such large size variations do not fit the multi-epoch data set, since the typical precision of the centro-nuclear radius estimate is $\lesssim 10 \%$. In addition, the flux dependence of $R_{\text {dust }}$ is constrained to $R \propto L^{(-0.3 \pm-0.3)}$ by the data. Thus, if indeed we would observe a variation of $R_{\text {dust }}$, then the trend would predict larger sizes at lower luminosity states, in contrast to an $L^{0.5}$ dependence.

However, the apparent slope can be explained by $F R_{\text {nuc }}^{K}$ variability alone, without the need for a variation of $R_{\text {dust }}$ between the three observations. Kishimoto et al. (2007) measured a significantly higher nuclear flux contribution to the $K$ band at a brighter state of the nucleus than during the observation of Riffel et al. (2009; 20\%-25\% with respect to $10 \%)$, although both teams fitted in comparable ways very similar blackbody temperatures to the NIR emission. The comparison of the two new precise visibility measurements reveal a small $V^{2}$ offset of 0.04 at the same angular resolution (compare the black and red data in Figure 1). Such a difference would result from changing $F R_{\text {nuc }}^{K}$ between both measurements from a $10 \%$ level in December 2008 to the $20 \%$ level in May 2009. The small $V^{2}$-difference, slightly larger than the conservative internight accuracy estimate of 0.03 , can be accommodated by a change of the compact $F R_{\text {nuc }}^{K}$, without further assumptions on the structure of the extended emission, in particular without changing the morphology of the extended brightness distribution. However, a change of the thermal flux by a factor of 2 , without changing the size of the radiating surface, would require a temperature change by $\sim 1.2$. As far as we know, such a luminosity-dependent dust temperature variation has not been observed yet in NGC 4151. In contrast, an increased radiation surface of a cloudy torus of fixed size would point to larger cloud covering factors or an increased number of efficiently heated clouds in brighter states.

Optical polarization and NIR-continuum variations appear correlated with visible nuclear continuum variations and enable reverberation experiments (see Gaskell et al. 2007, for a polarization reverberation experiment). Therefore, a variation of the fractional $F R_{\text {nuc }}^{K}$ cannot be explained by a varying line-ofsight extinction toward the nucleus (e.g., due to passing clouds), because such an apparent nuclear luminosity change should not result in the observed correlated change of optical polarization, and NIR-continuum fluxes. However, in a phase of nuclear brightening, the torus response might lag the nuclear flux rise by the reverberation delay. This could explain a momentary increase in $F R_{\text {nuc }}^{K}$ and in $V^{2}$, even if the intrinsic $R_{\text {dust }}$ does not change. 
Fifty milliparsecs equals 60 days of light travel, which matches the first four dust reverberation measurements of Koshida et al. (2009). Because these earlier dust reverberation measurements span already a factor of 6 in optical luminosity, they also suggest a luminosity-variation independent dust emission size. The first KI measurement by Swain et al. (2003) is quasi-contemporaneous with one of these dust reverberation measurements. Thus, both methods appear to agree very well. However, the former authors report on four subsequent, significantly smaller reverberation size estimates, the shortest being $\sim 30 \mathrm{mpc}$ only. Reverberation size estimates are biased to the innermost responding material (Gaskell \& Sparke 1986). Furthermore, if the responding structure is rather flat and inclined with respect to the line of sight, as expected for NGC 4151, the first response can set in considerable faster than light travel time corresponding to $R_{\text {dust }}$. However, in the case of an isotropic illumination, and a fixed dust geometry, such geometric effects could only explain a fix offset between reverberation and interferometric radii.

This discussion implies that dust reverberation sizes may often underestimate the location of the bulk of the circumnuclear dust, which dominates the $K$-band emission. Indeed, if we use our $R_{\text {dust }}$ for NGC 4151 , at least three of the four interferometric $R_{\text {dust }}$ estimates of the AGN, observed by Kishimoto et al. (2009a), exceed the $L^{0.5}$ fit of the reverberation size by factors of about $2 \pm 0.5$. The fourth object, Mrk 231 , likely has an inclined geometry, and larger, deprojected $R_{\text {dust }}$ as well (Smith et al. 2004). The direct interferometric $R_{\text {dust }}$ estimate appears as the more robust measurement of the average location of the bulk of the warm circum-nuclear dust (if projection ambiguities are well constrained). In contrast, the smallest reverberation sizes are smaller than the interferometric sizes, and the reverberation sizes vary beyond the scatter of the interferometric size estimates. We suggest that the reverberation sizes are probably tracing the location of momentary illuminated innermost, very hot dust clouds, instead of the bulk of the $K$ band dust. If circum-nuclear dust originates in outflows from the central engine (Elitzur \& Shlosman 2006, and references therein), then the shortest dust reverberation times could derive from optically thick dust in such outflows inside $R_{\text {dust }}$.

\section{CONCLUSIONS}

The results of a new interferometric NIR observing campaign of NGC 4151 are presented. Although this time in a 2 times fainter state, we managed to re-observe the AGN with the KI thanks to recent sensitivity improvements of the instrument. The interferometric visibilities and size estimates were compared to previously published interferometric and single telescope data. The current KI data sets suggest that the major part of the radiation originates in a (possibly inclined) toroidal structure of an intrinsic, deprojected radius of about $55 \pm 5 \mathrm{mpc}$, comparable to $V$-to- $K$-continuum reverberation measurements, without constraining the morphology in further detail.

Our data set and its comparison to published data enables for the first time the study of the sensitivity and response of near-infrared interferometric visibilities of an AGN to intrinsic flux variations. The observations show that NIR interferometry on bright AGNs $(K \sim 10 \mathrm{mag})$ are now feasible at a high precision of a few percent. We did not detect a significant visibility dependence on doubling the $K$-band emission of NGC 4151. This supports the notion, that, for an individual AGN, the size of its circum-nuclear NIR emission structure does not strictly depend on the respective momentary nuclear luminosity. The direct interferometric size estimates appear to be more robust estimates of the average location of the dust than NIR continuum reverberation estimates. Being flux independent, this average location of the hot dust probably relates to the sublimation radii of the AGN at its high activity state. Future dust reverberation campaigns, contemporaneous to direct interferometric measurements are highly desirable to study the circum-nuclear NIR emission region, to correctly interpret the reverberation measurements, and to investigate apparent changes in the dust illumination, heating efficiency, or covering factors.

We are grateful to the excellent KI team at WMKO and NExScI for making these observations a success. K. Meisenheimer and L. Burtscher contributed helpful discussions. The data presented herein were obtained at the W. M. Keck Observatory, which is operated as a scientific partnership among the California Institute of Technology, the University of California and the National Aeronautics and Space Administration. The Observatory was made possible by the generous financial support of the W. M. Keck Foundation. The authors recognize and acknowledge the very significant cultural role and reverence that the summit of Mauna Kea has always had within the indigenous Hawaiian community. We are most fortunate to have the opportunity to conduct observations from this mountain. The Keck Interferometer is funded by the National Aeronautics and Space Administration as part of its Navigator program. This work has made use of services produced by the NASA Exoplanet Science Institute at the California Institute of Technology. This research has made use of the SIMBAD database, operated at CDS, Strasbourg, France. This research has made use of the NASA/IPAC Extragalactic Database (NED) which is operated by the Jet Propulsion Laboratory, California Institute of Technology, under contract with the National Aeronautics and Space Administration.

Facilities: Keck:I, Keck:II

\section{REFERENCES}

Barvainis, R. 1987, ApJ, 320, 537

Barvainis, R. 1992, ApJ, 400, 502

Bentz, M. C., Peterson, B. M., Pogge, R. W., Vestergaard, M., \& Onken, C. A. 2006, ApJ, 644, 133

Burtscher, L., Jaffe, W., Raban, D., Meisenheimer, K., Tristram, K. R. W., \& Röttgering, H. 2009, ApJ, 705, L53

Colavita, M., et al. 2003, ApJ, 592, L83

Das, V., et al. 2005, AJ, 130, 945

Edelson, R. A., Gear, W. K. P., Malkan, M. A., \& Robson, E. I. 1988, Nature, 336, 749

Edelson, R. A., \& Malkan, M. A. 1986, ApJ, 308, 59

Elitzur, M., \& Shlosman, I. 2006, ApJ, 648, L101

Gaskell, C. M., Goosmann, R. W., Merkulova, N. I., Shakhovskoy, N. M., \& Shoji, M. 2007, arXiv:0711.1019

Gaskell, C. M., \& Sparke, L. S. 1986, ApJ, 305, 175

Hönig, S. F., Beckert, T., Ohnaka, K., \& Weigelt, G. 2006, A\&A, 452, 459

Hönig, S. F., \& Kishimoto, M. 2009, arXiv:0909.4539

Jaffe, W., et al. 2004, Nature, 429, 47

Kishimoto, M., Hönig, S. F., Antonucci, R., Kotani, T., Barvainis, R., Tristram, K. R. W., \& Weigelt, G. 2009a, A\&A, 507, L57

Kishimoto, M., Hönig, S. F., Beckert, T., \& Weigelt, G. 2007, A\&A, 476, 713

Kishimoto, M., Hönig, S. F., Tristram, K. R. W., \& Weigelt, G. 2009b, A\&A, 493, L57

Kobayashi, Y., Sato, S., Yamashita, T., Shiba, H., \& Takami, H. 1993, ApJ, 404, 94

Koshida, S., et al. 2009, ApJ, 700, L109

Lacy, J. H., et al. 1982, ApJ, 256, 75

Mor, R., Netzer, H., \& Elitzur, M. 2009, ApJ, 705, 298

Mundell, C. G., Wrobel, J. M., Pedlar, A., \& Gallimore, J. F. 2003, ApJ, 583, 192 
Nenkova, M., Sirocky, M. M., Ivezić, Ž., \& Elitzur, M. 2008a, ApJ, 685, 147

Nenkova, M., Sirocky, M. M., Nikutta, R., Ivezić, Ž., \& Elitzur, M. 2008b, ApJ, 685,160

Pacholczyk, A. G., \& Weymann, R. J. 1968, AJ, 73, 870

Raban, D., Jaffe, W., Röttgering, H., Meisenheimer, K., \& Tristram, K. R. W. 2009, MNRAS, 394, 1325

Ragland, S., et al. 2008, Proc. SPIE, 7013, 10

Rees, M. J., Silk, J. I., Werner, M. W., \& Wickramasinghe, N. C. 1969, Nature, 223,788

Riffel, R., Rodríguez-Ardila, A., \& Pastoriza, M. G. 2006, A\&A, 457, 61

Riffel, R. A., Storchi-Bergmann, T., \& McGregor, P. J. 2009, ApJ, 698, 1767
Sanders, D. B., Phinney, E. S., Neugebauer, G., Soifer, B. T., \& Matthews, K. 1989, ApJ, 347, 29

Schartmann, M., Meisenheimer, K., Camenzind, M., Wolf, S., \& Henning, T. 2005, A\&A, 437, 861

Shapovalova, A. I., et al. 2008, A\&A, 486, 99

Smith, J. E., Robinson, A., Alexander, D. M., Young, S., Axon, D. J., \& Corbett, E. A. 2004, MNRAS, 350, 140

Suganuma, M., et al. 2006, ApJ, 639, 46

Swain, M., et al. 2003, ApJ, 596, L163

Tristram, K. R. W., et al. 2009, A\&A, 502, 67

Wittkowski, M., Kervella, P., Arsenault, R., Paresce, F., Beckert, T., \& Weigelt, G. 2004, A\&A, 418, L39

Wizinowich, P., et al. 2006, Proc. SPIE, 6268, 21 